\title{
Evolution of the First Eigenvalue of a $(p, q)$-Laplacian Under a Harmonic Ricci Flow
}

\author{
Paul Bracken \\ Department of Mathematics, University of Texas, Edinburg, TX, USA \\ Email: paul.bracken@utrgv.edu
}

How to cite this paper: Bracken, P. (2021) Evolution of the First Eigenvalue of a $(p, q)$-Laplacian Under a Harmonic Ricci Flow. Advances in Pure Mathematics, 11, 205-217.

https://doi.org/10.4236/apm.2021.114015

Received: March 1, 2021

Accepted: April 3, 2021

Published: April 6, 2021

Copyright (c) 2021 by author(s) and Scientific Research Publishing Inc. This work is licensed under the Creative Commons Attribution International License (CC BY 4.0).

http://creativecommons.org/licenses/by/4.0/

\begin{abstract}
The properties of the first eigenvalue of a class of $(p, q)$ Laplacian are investigated. A variational formulation for the first eigenvalue of the Laplacian on a closed Riemannian manifold is obtained. This eigenvalue corresponds to a nonlinear, coupled system of $p$-Laplacian partial differential equations. The main idea is to investigate the evolution of the first eigenvalue of the system under the Ricci harmonic flow. It is also possible to construct monotonic quantities based on them and study their evolution which is done.
\end{abstract}

\section{Keywords}

Ricci Flow, Curvature, Eigenvalue, Evolution, Laplacian, Nonlinear

\section{Introduction}

The eigenvalue problem which arises from geometric operators under various kinds of geometric flows has attracted a great deal of attention recently, as it is a very effective method of studying Riemannian manifolds. This area of investigation opened up when Perlman [1] showed that a functional depending on scalar curvature is nondecreasing along the Ricci flow coupled to a type of heat equation [2] [3]. This property of the functional implies that the first eigenvalue of a geometric operator is nondecreasing under Ricci flow. The geometric operator $-\Delta+c R$ has also been studied with regard to its eigenvalues along the Ricci flow and Ricci-Bourguignon flow as well. The evolution of the first eigenvalue has been looked at in the case of the $p$-Laplacian along a Ricci-harmonic flow, and the Ricci flow and the $m$-th mean curvature flow respectively [4] [5]. There is a generalization of the $p$-Laplacian to a class of $(p, q)$-Laplacian which has applications in applied mathematics and physics [6] [7].

A geometric flow is an evolution of a geometric structure relevant to a given 
manifold. Let $M^{m}$ be a closed, $m$-dimensional Riemannian manifold that has metric $g$. Hamilton first introduced the Ricci flow by means of the differential equation

$$
\frac{\partial g(t)}{\partial t}=-\operatorname{Ric}(g(t)), \quad g(0)=g_{0} \text {. }
$$

In (1), $t$ is an evolution parameter and Ric is the Ricci tensor of metric $g(t)$. Lowered indices are understood to apply in (1) so that $g(t)=g_{i j}(t)$. Let $\left(M^{m}, g\right)$ and $\left(N^{n}, \sigma\right)$ be two closed Riemannian manifolds. By Nash's embedding theorem, it may be assumed that $N^{n}$ is isometrically embedded into Euclidean space $i_{N}:\left(N^{n}, \sigma\right) \rightarrow \mathbb{R}^{n+1}$. Identify maps $\varphi: M^{m} \rightarrow N^{n}$ with $i_{N} \circ \varphi: M^{m} \rightarrow \mathbb{R}^{d}$ for sufficiently large $d$. Then a generalization of Ricci flow can be established as follows,

$$
\begin{aligned}
& \frac{\partial g(t)}{\partial t}=-2 \operatorname{Ric}(g(t))+2 \alpha \nabla \varphi \otimes \nabla \varphi, \quad g(0)=g_{0}, \\
& \frac{\partial \varphi}{\partial t}=\mathcal{L}_{g} \varphi, \quad \varphi(0)=\varphi_{0} .
\end{aligned}
$$

In (2), $\alpha$ is a positive constant, $\varphi(t)$ is a family of smooth maps from $M^{m}$ to a closed target manifold $N^{n}$ and $\mathcal{L} \varphi$ is the intrinsic Laplacian of $\varphi$ which denotes the tension field of $\varphi$ with respect to the evolving metric $g(t)$. This system of evolution equations will be called the Ricci flow coupled to a harmonic flow. It has been shown that (2) has a unique solution with the initial data $\left(g_{0}, \varphi_{0}\right)$. It is also useful to define a normalized Ricci-harmonic flow defined as

$$
\begin{aligned}
& \frac{\partial g(t)}{\partial t}=-2 \operatorname{Ric}(g(t))+2 \alpha \nabla \varphi \otimes \nabla \varphi+\frac{2}{m} r(t) g(t), \quad g(0)=g_{0}, \\
& \frac{\partial \varphi}{\partial t}=\mathcal{L}_{g} \varphi, \quad \varphi(0)=\varphi_{0} .
\end{aligned}
$$

The variable $r(t)$ in (3) is called the average of $R-\alpha|\nabla \varphi|^{2}$ with respect to $M^{m}$, and defined as

$$
r=\frac{\int_{M}\left(R-\alpha|\nabla \varphi|^{2}\right) \mathrm{d} \mu}{\int_{M} \mathrm{~d} \mu}
$$

When integrating over the manifold, we simply write $M$, and $\mathrm{d} \mu$ is the volume form or measure on $M^{m}$. Under normalized Ricci flow, the volume of the solution metrics remains constant with respect to $t$.

\section{Definition of the Eigenvalue Problem}

Let $\left(M^{m}, g\right)$ be a closed Riemannian manifold and let $f: M^{m} \rightarrow \mathbb{R}$ be a smooth function on the manifold so suppose $f \in W^{1, p}(M)$. The Laplace-Beltrami operator which acts on a smooth function $f$ defined on $M^{m}$ is the divergence of the gradient of $f$, 


$$
\Delta f=\frac{1}{\sqrt{\operatorname{det}(g)}} \partial_{i}\left(\sqrt{\operatorname{det} g} \partial_{j} f\right) .
$$

where we have set $\partial_{i} f=\partial f / \partial x^{i}$. The $p$-Laplacian of $f$ is defined for $1<p<\infty$ as

$$
\Delta_{p} f=\operatorname{div}\left(|\nabla f|^{p-2} \nabla f\right)=|\nabla f|^{p-2} \Delta f+(p-2)|\nabla f|^{p-4}(\text { Hess } f)(\nabla f, \nabla f),
$$

where $($ Hess $f)(X, Y)=\nabla(\nabla f)(X, Y)$, where $X, Y$ are vector fields on $M^{m}$. In local coordinates,

$$
(\operatorname{Hess} f)\left(\partial_{i}, \partial_{j}\right)=\partial_{i} \partial_{j} f-\Gamma_{i j}^{k} \partial_{k} f .
$$

When $p=2$ the $p$-Laplacian becomes the Laplace-Beltrami operator. Let $\left(M^{m}, g\right)$ be a closed Riemannian manifold. To present the problem, consider the following nonlinear system of equations on $M^{m}$

$$
\begin{aligned}
& \Delta_{p} u=\lambda|u|^{\alpha}|v|^{\beta} v, \\
& \Delta_{p} v=-\lambda|u|^{\alpha}|v|^{\beta} u, \\
& u, v \in W^{1, p}(M) \times W^{1, q}(M) .
\end{aligned}
$$

In (8), $p>1$ and $q>1$ and $\alpha, \beta$ are positive real numbers which satisfy the condition

$$
\frac{\alpha+1}{p}+\frac{\beta+1}{q}=1
$$

It is said that $\lambda$ in (8) is an eigenvalue for the system whenever for some $u \in W_{0}^{1, p}(M)$ and $v \in W_{0}^{1, q}(M)$ it is the case that

$$
\begin{aligned}
& \int_{M}|\nabla u|^{p-2}\langle\nabla u, \nabla \phi\rangle \mathrm{d} \mu=\lambda \int_{M}|u|^{\alpha}|v|^{\beta} v \phi \mathrm{d} \mu, \\
& \int_{M}|\nabla v|^{q-2}\langle\nabla v, \nabla \psi\rangle \mathrm{d} \mu=\lambda \int_{M}|u|^{\alpha}|v|^{\beta} u \psi \mathrm{d} \mu .
\end{aligned}
$$

The functions $\phi \in W^{1, p}(M), \psi \in W^{1, q}(M)$ and $W_{0}^{1, p}(M)$ is the closure of $C_{0}^{\infty}(M)$ in the Sobolev space $W^{1, p}(M)$. The set of functions $u, v$ are called the eigenfunctions which correspond to the eigenvalue $\lambda$. A first positive eigenvalue of (8) can be determined by computing

$$
\inf \left\{A(u, v):(u, v) \in W_{0}^{1, p}(M) \times W^{1, q}(M), B(u, v)=1\right\} .
$$

In (11), $A(u, v)$ and $B(u, v)$ are defined to be

$$
\begin{aligned}
& A(u, v)=\frac{\alpha+1}{p} \int_{M}|\nabla u|^{p} \mathrm{~d} \mu+\frac{\beta+1}{q} \int_{M}|\nabla v|^{q} \mathrm{~d} \mu, \\
& B(u, v)=\int_{M}|u|^{\alpha}|v|^{\beta} u v \mathrm{~d} \mu .
\end{aligned}
$$

Let $\left(M^{m}, g(t), \varphi(t)\right)$ be a solution of the $R H^{\alpha}$ flow (1) on the smooth manifold $\left(M^{m}, g_{0}, \varphi_{0}\right)$ with $t \in[0, T)$. Then

$$
\lambda(t)=\frac{\alpha+1}{p} \int_{M}|\nabla u|^{p} \mathrm{~d} \mu+\frac{\beta+1}{q} \int_{M}|\nabla v|^{q} \mathrm{~d} \mu
$$

defines the solution of an eigenvalue of (8) under the variation of $(g(t), \varphi(t))$. 
The eigenfunctions associated to $\lambda(t)$ are normalized such that $B(u, v)=1$.

The first eigenvalue of a class of $(p, q)$-Laplacians given in (8) is studied such that its metric satisfies the flow. Let us denote differentiation with respect to $t$ as $\partial u / \partial t=\partial_{t} u=u^{\prime}$, and introduce tensor $\mathcal{S}$ and its trace

$$
\mathcal{S}=\text { Ric }_{g}-\alpha \nabla \varphi \otimes \nabla \varphi, \quad S=R-\alpha|\nabla \varphi|^{2},
$$

where $R$ is the Ricci scalar curvature.

\section{Variational Formulation}

Some useful evolution equations for $\lambda(t)$ under the Ricci harmonic flow will be formulated. In particular, a useful result concerning the variation of the first eigenvalue (8) under the Ricci harmonic flow is considered next.

Theorem 1: Let $(g(t), \varphi(t))$ with $t \in[0, T)$ be a solution of the Ricci harmonic flow on the closed manifold $M^{m}$. Let $\lambda(t)$ be the first eigenvalue of the $(p, q)$-Laplacian along this flow. For any $t_{0}, t_{1} \in[0, T)$ such that $t_{1}>t_{0}$, we have

$$
\lambda\left(t_{1}\right) \geq \lambda\left(t_{0}\right)+\int_{t_{0}}^{t_{1}} \mathcal{G}(g(\tau), u(\tau), v(\tau)) \mathrm{d} \tau .
$$

The integrand is given by

$$
\begin{aligned}
\mathcal{G}(g(t), u(t), v(t))= & (\alpha+1) \int_{M}\left(\mathcal{S}(\nabla u, \nabla u)+\left\langle\nabla u^{\prime}, \nabla u\right\rangle\right)|\nabla u|^{p-2} \mathrm{~d} \mu \\
& +(\beta+1) \int_{M}\left(\mathcal{S}(\nabla v, \nabla v)+\left\langle\nabla v^{\prime}, \nabla v\right\rangle\right)|\nabla v|^{q-2} \mathrm{~d} \mu \\
& -\frac{\alpha+1}{p} \int_{M}|\nabla u|^{p} \mathrm{~d} \mu-\frac{\beta+1}{q} \int_{M}|\nabla v|^{q} \mathrm{~d} \mu .
\end{aligned}
$$

Proof: Let us put

$$
G(g(t), u(t), v(t))=\frac{\alpha+1}{p} \int_{M}|\nabla u(t)|_{g(t)}^{p} \mathrm{~d} \mu+\frac{\beta+1}{q} \int_{M}|\nabla v(t)|_{g(t)}^{q} \mathrm{~d} \mu_{g(t)} .
$$

suppose that at $t_{1}$ we assign $\left(u_{1}, v_{1}\right)=\left(u\left(t_{1}\right), v\left(t_{1}\right)\right)$ be the eigenfunctions corresponding to the eigenvalue $\lambda\left(t_{1}\right)$ for the $(p, q)$-Laplacian. Define the following smooth functions along the Ricci harmonic flow as follows,

$$
h(t)=u_{1}\left(\frac{\operatorname{det}\left(g_{i j}\left(t_{1}\right)\right)}{\operatorname{det}\left(g_{i j}(t)\right)}\right)^{1 /(2(\alpha+\beta+1))}, \quad k(t)=v_{1}\left(\frac{\operatorname{det}\left(g_{i j}\left(t_{1}\right)\right)}{\operatorname{det}\left(g_{i j}(t)\right)}\right)^{1 /(2(\alpha+\beta+1))} .
$$

Furthermore, functions $u, v$ can be defined along this flow according to the equations

$$
\begin{aligned}
& u(t)=\frac{h(t)}{\left(\int_{M}|h(t)|^{\alpha}|k(t)|^{\beta} h(t) k(t) \mathrm{d} \mu\right)^{1 / p}}, \\
& v(t)=\frac{k(t)}{\left(\int_{M}|h(t)|^{\alpha}|k(t)|^{\beta} h(t) k(t) \mathrm{d} \mu\right)^{1 / q}} .
\end{aligned}
$$

In (19), the functions $u(t)$ and $v(t)$ are smooth functions under the Ricci harmonic flow and they satisfy the condition 


$$
\int_{M}|u|^{\alpha}|v|^{\beta} u v \mathrm{~d} \mu=1 .
$$

Now $\left(u\left(t_{1}\right), v\left(t_{1}\right)\right)$ are the eigenfunctions of the eigenvalue $\lambda\left(t_{1}\right)$ for the $(p, q)$-Laplacian at time $t_{1}$, that is, $\lambda\left(t_{1}\right)=G\left(g\left(t_{1}\right), u\left(t_{1}\right), v\left(t_{1}\right)\right)$. The following formula will be needed, which arises from the fact that

$$
g_{i j} \frac{\partial g^{j k}}{\partial t}=-\frac{\partial g_{i j}}{\partial t} g^{j k}
$$

With (21), (3) can be expressed in components using (14) as

$$
\frac{\partial g^{l k}}{\partial t}=-g^{l i} g^{j k} \frac{\partial g_{i j}}{\partial t}=2 g^{l i} g^{j k} \mathcal{S}_{i j} .
$$

Hence, if $f$ is a smooth function with respect to $t$, then along the Ricci-harmonic flow, we find that

$$
\frac{\mathrm{d}}{\mathrm{d} t}\left(|\nabla f|^{p}\right)=\frac{p}{2}\left(\frac{\partial}{\partial t} g^{i j} \nabla_{i} f \nabla_{j} f+2 g^{i j} \nabla_{i} f^{\prime} \nabla_{j} f\right)|\nabla f|^{p-2} .
$$

Substituting the result from (22), we have

$$
\frac{\mathrm{d}}{\mathrm{d} t}\left(|\nabla f|^{p}\right)=p|\nabla f|^{p-2}\left(\mathcal{S}(\nabla f, \nabla)+\left\langle\nabla f^{\prime}, \nabla f\right\rangle\right)
$$

The measure $\mathrm{d} \mu$ also depends on $t$ through $g$ and has derivative

$$
\frac{\partial}{\partial t} \mathrm{~d} \mu=\frac{1}{2} \operatorname{Tr}_{g}\left(\frac{\partial g}{\partial t}\right) \mathrm{d} \mu=-S \mathrm{~d} \mu .
$$

Since $u(t)$ and $v(t)$ are smooth functions so too is $G(g(t), u(t), v(t))$ with respect to $t$. Let us write

$$
\mathcal{G}(g(t), u(t), v(t))=\frac{\mathrm{d}}{\mathrm{d} t} G(g(t), u(t), v(t)) .
$$

Using (24) and (25) with freplaced by $u$ and $v$, it follows that,

$$
\begin{aligned}
\mathcal{G}(g(t), u(t), v(t))= & (\alpha+1) \int_{M}\left(\mathcal{S}(\nabla u, \nabla u)+\left\langle\nabla u^{\prime}, \nabla u\right\rangle\right)|\nabla u|^{p-2} \mathrm{~d} \mu \\
& +(\beta+1) \int_{M}\left(\mathcal{S}(\nabla v, \nabla v)+\left\langle\nabla v^{\prime}, \nabla v\right\rangle\right)|\nabla v|^{q-2} \mathrm{~d} \mu \\
& -\frac{\alpha+1}{p} \int_{M}|\nabla u|^{p} S \mathrm{~d} \mu-\frac{\beta+1}{q} \int_{M}|\nabla v|^{q} S \mathrm{~d} \mu .
\end{aligned}
$$

Integrating both sides of (26) with respect to $t$ between $t_{0}$ and $t_{1}$, it follows that

$$
\int_{t_{0}}^{t_{1}} \mathcal{G}(g(\tau), u(\tau), v(\tau)) \mathrm{d} \tau=G\left(g\left(t_{1}\right), u\left(t_{1}\right), v\left(t_{1}\right)\right)-G\left(g\left(t_{0}\right), u\left(t_{0}\right), v\left(t_{0}\right)\right),
$$

where $t_{0} \in[0, T)$ and $t_{1}>t_{0}$. since it is the case that $G\left(g\left(t_{0}\right), u\left(t_{0}\right), v\left(t_{0}\right)\right) \geq \lambda\left(t_{0}\right)$, then setting $\lambda\left(t_{1}\right)=G\left(g\left(t_{1}\right), u\left(t_{1}\right), v\left(t_{1}\right)\right)$ in (28), it is seen that (15) follows immediately with $S(g(t), u(t), v(t))$ satisfying (16).

Theorem 2: Let $\left(M^{m}, g(t), \varphi(t)\right)$ be a solution of the Ricci-harmonic flow on the smooth, closed manifold $\left(M^{m}, g_{0}, \varphi_{0}\right)$ and let $\lambda(t)$ denote the evolu- 
tion of the first eigenvalue under the flow. Suppose that $\kappa=\min \{p, q\}$ and on $M^{m} \times[0, T)$ it holds that

$$
\mathcal{S}-\frac{1}{\kappa} S g \geq 0 .
$$

If $S_{\min }(0) \geq 0$ then $\lambda(t)$ is nondecreasing and differentiable almost everywhere along the Ricci-harmonic flow (2) on $[0, T)$.

Proof: For any $t_{1} \in[0, T)$ let $u\left(t_{1}\right), v\left(t_{1}\right)$ be the eigenfunctions corresponding to the value $\lambda\left(t_{1}\right)$ of the $(p, q)$-Laplacian. Then there is the normalization condition

$$
\int_{M}\left|u\left(t_{1}\right)\right|^{\alpha}\left|v\left(t_{1}\right)\right|^{\beta} u\left(t_{1}\right) v\left(t_{1}\right) \mathrm{d} \mu_{g(t)}=1 .
$$

Thus (16) is given by

$$
\begin{aligned}
\mathcal{G}\left(g\left(t_{1}\right), u\left(t_{1}\right), v\left(t_{1}\right)\right)= & (\alpha+1) \int_{M}\left(\mathcal{S}(\nabla u, \nabla u)+\left\langle\nabla u^{\prime}, \nabla u\right\rangle\right)|\nabla u|^{p-2} \mathrm{~d} \mu \\
& +(\beta+1) \int_{M}\left(\mathcal{S}(\nabla v, \nabla v)+\left\langle\nabla v^{\prime}, \nabla v\right\rangle\right)|\nabla v|^{q-2} \mathrm{~d} \mu \\
& -\frac{\alpha+1}{p} \int_{M}|\nabla u|^{p} \mathrm{~d} \mu-\frac{\beta+1}{q} \int_{M}|\nabla v|^{q} \mathrm{~d} \mu .
\end{aligned}
$$

Differentiating the normalization condition and using (25), we get

$$
(\alpha+1) \int_{M}|u|^{\alpha}|v|^{\beta} u^{\prime} v \mathrm{~d} \mu+(\beta+1) \int_{M}|u|^{\alpha}|v|^{\beta} u v^{\prime} \mathrm{d} \mu-\int_{M}|u|^{\alpha}|v|^{\beta} u v \mathrm{~d} \mu=0 .
$$

The results in (10) imply that by replacing function $\phi$ by $u^{\prime}$ and $\psi$ by $v^{\prime}$, one obtains

$$
\begin{aligned}
& \int_{M}\left\langle\nabla u^{\prime}, \nabla u\right\rangle|\nabla u|^{p-2} \mathrm{~d} \mu=\lambda\left(t_{1}\right) \int_{M}|u|^{\alpha}|v|^{\beta} u^{\prime} v \mathrm{~d} \mu, \\
& \int_{M}\left\langle\nabla v^{\prime}, \nabla v\right\rangle|\nabla v|^{q-2} \mathrm{~d} \mu=\lambda\left(t_{1}\right) \int_{M}|u|^{\alpha}|v|^{\beta} u v \mathrm{~d} \mu .
\end{aligned}
$$

Multiply the first equation in (33) by $\alpha+1$ and the second by $\beta+1$ and then add the two, then we obtain that

$$
\begin{aligned}
& \lambda\left(t_{1}\right)(\alpha+1) \int_{M}|u|^{\alpha}|v|^{\beta} u^{\prime} v \mathrm{~d} \mu+\lambda\left(t_{1}\right)(\beta+1) \int_{M}|u|^{\alpha}|v|^{\beta} u v^{\prime} \mathrm{d} \mu \\
& =(\alpha+1) \int_{M}\left\langle\nabla u^{\prime}, \nabla u\right\rangle|\nabla u|^{p-2} \mathrm{~d} \mu+(\beta+1) \int_{M}\left\langle\nabla v^{\prime}, \nabla v\right\rangle|\nabla v|^{q-2} \mathrm{~d} \mu .
\end{aligned}
$$

Multiply (32) by $\lambda\left(t_{1}\right)$ and then subtract the resulting expression from (34),

$$
\begin{aligned}
& (\alpha+1) \int_{M}\left\langle\nabla u^{\prime}, \nabla u\right\rangle|\nabla u|^{p-2} \mathrm{~d} \mu+(\beta+1) \int_{M}\left\langle\nabla v^{\prime}, \nabla v\right\rangle|\nabla v|^{q-2} \mathrm{~d} \mu \\
& -\lambda\left(t_{1}\right) \int_{M}|u|^{\alpha}|v|^{\beta} S u v \mathrm{~d} \mu=0 .
\end{aligned}
$$

Substituting (35) into $\mathcal{G}$ given in (31), we have

$$
\begin{aligned}
\mathcal{G}(g(t), u(t), v(t))= & \lambda\left(t_{1}\right) \int_{M} S|u|^{\alpha}|v|^{\beta} u v \mathrm{~d} \mu \\
& +(\alpha+1) \int_{M} \mathcal{S}(\nabla u, \nabla u)|\nabla u|^{p-2} \mathrm{~d} \mu-\frac{\alpha+1}{p} \int_{M}|\nabla u|^{p} S \mathrm{~d} \mu \\
& +(\beta+1) \int_{M} \mathcal{S}(\nabla v, \nabla v)|\nabla v|^{q-2} \mathrm{~d} \mu+\frac{\beta+1}{q} \int_{M}|\nabla v|^{q} \mathrm{~d} \mu .
\end{aligned}
$$

Substitute the hypothesis given in (29) into (36) to yield the inequality 


$$
\begin{aligned}
\mathcal{G} & \left.g\left(t_{1}\right), u\left(t_{1}\right), v\left(t_{1}\right)\right) \\
\geq & \lambda\left(t_{1}\right) \int_{M} S|u|^{\alpha}|v|^{\beta} u v \mathrm{~d} \mu+\frac{\alpha+1}{\kappa} \int_{M} S g^{i j} \nabla_{i} u \nabla_{j} u|\nabla u|^{p-2} \mathrm{~d} \mu \\
& -\frac{\alpha+1}{p} \int_{M}|\nabla u|^{p} S \mathrm{~d} \mu+\frac{p+1}{\kappa} \int_{M} S g^{i j} \nabla_{i} v \nabla_{j} v|\nabla v|^{q-2} \mathrm{~d} \mu-\frac{p+1}{q} \int_{M}|\nabla v|^{q} S \mathrm{~d} \mu \\
= & \lambda\left(t_{1}\right) \int_{M} S|u|^{\alpha}|v|^{\beta} u v \mathrm{~d} \mu+(\alpha+1)\left(\frac{1}{\kappa}-\frac{1}{p}\right) \int_{M}|\nabla u|^{p} S \mathrm{~d} \mu \\
& +(\beta+1)\left(\frac{1}{\kappa}-\frac{1}{q}\right) \int_{M}|\nabla v|^{q} \mathrm{~d} \mu .
\end{aligned}
$$

Using the definition of $\mathcal{S}$ from (14) and the two known results

$$
\frac{\partial S}{\partial t}=\Delta S+2\left|\mathcal{S}_{i j}\right|^{2}+2 \alpha\left|\mathcal{L}_{g} \varphi\right|^{2}, \quad\left|\mathcal{S}_{i j}\right| \geq \frac{1}{m} S^{2},
$$

it follows that since $\alpha>0$ the last term in (38) is positive the lower bound results

$$
\frac{\partial S}{\partial t} \geq \Delta S+\frac{2}{m} S^{2},
$$

Thus $S$ is a supersolution of the partial differential equation $\partial Q / \partial t=\Delta Q+(2 / m) Q^{2}$. To be able to use the maximum principle, it has to be observed that the solution to the equation

$$
\frac{\mathrm{d} y(t)}{\mathrm{d} t}=\frac{2}{m} y(t)^{2}, \quad y(0)=S_{\min }(0),
$$

is exactly the function

$$
y(t)=\frac{S_{\min }(0)}{1-\frac{2}{m} S_{\min }(0) t},
$$

for $t \in\left[0, T^{\prime}\right)$, where $T^{\prime}=\min \left\{T, m / 2 S_{\text {min }}(0)\right\}$. Applying the maximum principle to (39), it must be that $S=y(t)$ along the Ricci-harmonic flow. If $S_{\text {min }}(0) \geq 0$ the nonnegativity of $S$ is preserved along the flow and (37) has the property,

$$
\mathcal{G}\left(g\left(t_{1}\right), u\left(t_{1}\right), v\left(t_{1}\right)\right) \geq 0 .
$$

In any small neighborhood of $t_{1}$ then it also holds that $\mathcal{G}(g(t), u(t), v(t)) \geq 0$. So it follows that for any $t_{0}<t_{1}$ sufficiently close to $t_{1}$,

$$
\int_{t_{0}}^{t_{1}} \mathcal{G}(g(\tau), u(\tau), v(\tau)) \mathrm{d} \tau>0 .
$$

Since $t_{1} \in[0, T)$ is arbitrary, the first part of the claim is complete. For differentiability of $\lambda(t)$ note that as $\lambda(t)$ is increasing and continuous on the interval $[0, T)$, the Lebesgue theorem implies that function $\lambda(t)$ is differentiable almost everywhere on $[0, T)$. Thus the proof is complete.

\section{Ricci Flows}

A smooth eigenvalue function can be introduced along the Ricci harmonic flow. 
Evolution equations can be developed for this. Let $M^{m}$ be an $m$-dimensional closed Riemannian manifold and let $g(t)$ be a smooth solution of the flow. Introduce a function which depends on $u, v$ and $g(t)$ which satisfy the three integral constraints

$$
\int_{M}|u|^{\alpha}|v|^{\beta} u v \mathrm{~d} \mu=1, \quad \int_{M}|u|^{\alpha}|v|^{\beta} v \mathrm{~d} \mu=0, \quad \int_{M}|u|^{\alpha}|v|^{\beta} u \mathrm{~d} \mu=0 .
$$

In terms of $u$ and $v$, let us introduce the function

$$
\lambda(u, v, t)=\frac{\alpha+1}{p} \int_{M}|\nabla u|^{p} \mathrm{~d} \mu+\frac{\beta+1}{q} \int_{M}|\nabla v|^{q} \mathrm{~d} \mu .
$$

With respect to the variable $t, \lambda(u, v, t)$ is a snooth eigenvalue type function. In the case where $u, v$ are the corresponding eigenfunctions corresponding to the first eigenvalue $\lambda\left(t_{1}\right)$, then $\lambda(u, v, t)=\lambda\left(t_{1}\right)$. In this case, (45) gives the eigenvalue directly without going through the process indicated in (11). This leads us to formulate the following Proposition which can be proved along exactly the same lines as the two proceeding results.

Proposition 1: Let $\left(M^{m}, g(t), \varphi(t)\right)$ be a solution of the Ricci harmonic flow on the smooth closed manifold $\left(M^{m}, g_{0}, \varphi_{0}\right)$. If $\lambda(t)$ denotes the evolution of the first eigenvalue under this flow, then

$$
\begin{aligned}
\frac{\mathrm{d} \lambda}{\mathrm{d} t}(u, v, t)= & \lambda\left(t_{1}\right) \int_{M} S|u|^{\alpha}|v|^{\beta} u v \mathrm{~d} \mu \\
& +(\alpha+1) \int_{M} \mathcal{S}(\nabla u, \nabla u)|\nabla u|^{p-2} \mathrm{~d} \mu-\frac{\alpha+1}{p} \int_{M}|\nabla u|^{p} S \mathrm{~d} \mu \\
& +(\beta+1) \int_{M} \mathcal{S}(\nabla v, \nabla v)|\nabla v|^{q-2} \mathrm{~d} \mu-\frac{\beta+1}{q} \int_{M}|\nabla v|^{q} S \mathrm{~d} \mu .
\end{aligned}
$$

Here $u$ and $v$ are the associated normalized evolving eigenfunctions.

At this point we can start to study the evolution of $\lambda(t)$ under the normalized flow (3), which is similar to what has already been done.

Theorem 3: Let $\left(M^{m}, g(t), \varphi(t)\right)$ be a solution of the normalized Ricci harmonic flow on a smooth closed manifold $\left(M^{m}, g_{0}, \varphi_{0}\right)$. If $\lambda(t)$ denotes the evolution of the first eigenvalue under the flow (3), then

$$
\begin{aligned}
\frac{\partial \lambda}{\partial t}(u, v, t)_{t=t_{1}}= & \lambda\left(t_{1}\right) \int_{M} S|u|^{\alpha}|v|^{\beta} u v \mathrm{~d} \mu+(\alpha+1) \int_{M} \mathcal{S}(\nabla u, \nabla u)|\nabla u|^{p-2} \mathrm{~d} \mu \\
& +(\beta+1) \int_{M} \mathcal{S}(\nabla v, \nabla v)|\nabla v|^{q-2} \mathrm{~d} \mu-\frac{\beta+1}{q} \int_{M}|\nabla v|^{q} S \mathrm{~d} \mu \\
& -\frac{\alpha+1}{p} \int_{M}|\nabla u|^{p} S \mathrm{~d} \mu-\frac{\alpha+1}{m} r\left(t_{1}\right) \int_{M}|\nabla u|^{p} \mathrm{~d} \mu \\
& -\frac{\beta+1}{m} r\left(t_{1}\right) \int_{M}|\nabla v|^{q} \mathrm{~d} \mu,
\end{aligned}
$$

in which $u, v$ are the associated evolving, normalized eigenfunctions for the problem.

Proof: In the normalized case start by differentiating the first integrability condition in (44) with respect to the parameter to find 


$$
\begin{aligned}
& (\alpha+1) \int_{M}|u|^{p}|v|^{q} u^{\prime} v \mathrm{~d} \mu+(\beta+1) \int_{M}|u|^{p}|v|^{q} u v^{\prime} \mathrm{d} \mu \\
& =-r(t)+\int_{M} S|u|^{q}|q|^{q} u v \mathrm{~d} \mu .
\end{aligned}
$$

To get the right-hand side, Equation (25) has to be modified to

$$
\frac{\partial}{\partial t} \mathrm{~d} \mu=\frac{1}{2} \operatorname{Tr}\left(\frac{\partial g}{\partial t}\right) \mathrm{d} \mu=\frac{1}{2} \operatorname{Tr}\left(-S_{i j}+\frac{2}{m} r g_{i j}\right) \mathrm{d} \mu=(r-S) \mathrm{d} \mu .
$$

Hence the $t$ derivative of $\lambda$ is given by

$$
\begin{aligned}
& \frac{\partial \lambda}{\partial t}(u, v, t)_{t=t_{1}} \\
& =\frac{\alpha+1}{p} \int_{M}\left(\frac{p}{2}|\nabla u|^{p-2}\left(2 \mathcal{S}(\nabla u, \nabla u)+2\left\langle\nabla u^{\prime}, \nabla u\right\rangle-\frac{2}{m} r\langle\nabla u, \nabla u\rangle\right)\right. \\
& \left.\quad+|\nabla u|^{p}(r-S)\right) \mathrm{d} \mu \\
& \quad+\frac{\beta+1}{q} \int_{M}\left(\frac{q}{2}|\nabla v|^{q-2}\left(2 \mathcal{S}(\nabla v, \nabla v)+2\left\langle\nabla v^{\prime}, \nabla v\right\rangle+|\nabla v|^{q}(r-S)\right) \mathrm{d} \mu .\right.
\end{aligned}
$$

For the normalized Ricci flow, the following relation holds,

$$
\begin{aligned}
& (\alpha+1) \int_{M}\left\langle\nabla u^{\prime}, \nabla u\right\rangle|\nabla u|^{p-2} \mathrm{~d} \mu+(\beta+1) \int_{M}\left\langle\nabla v^{\prime}, \nabla v\right\rangle|\nabla v|^{q} \mathrm{~d} \mu \\
& =-\lambda\left(t_{1}\right) r\left(t_{1}\right)+\lambda\left(t_{1}\right) \int_{M} S|u|^{\alpha}|v|^{\beta} u v \mathrm{~d} \mu .
\end{aligned}
$$

Now replacing (51) in (50), we obtain the result

$$
\begin{aligned}
\left.\frac{\partial \lambda}{\partial t}\right|_{t=t_{1}} & =(\alpha+1) \int_{M}\left(\mathcal{S}(\nabla u, \nabla u)-\frac{r}{m}|\nabla u|^{2}\right)|u|^{p-2} \mathrm{~d} \mu-\frac{\alpha+1}{p} \int_{M} S|\nabla u|^{p} \mathrm{~d} \mu \\
& +(\beta+1) \int_{M}\left(\mathcal{S}(\nabla v, \nabla v)-\frac{r}{m}|\nabla v|^{2}\right)|\nabla v|^{q-2} \mathrm{~d} \mu-\frac{\beta+1}{q} \int_{M} S|\nabla v|^{q} \mathrm{~d} \mu \\
& +r\left(t_{1}\right)\left(\frac{\alpha+1}{p} \int_{M}|\nabla u|^{p} \mathrm{~d} \mu+\frac{\beta+1}{q} \int_{M}|\nabla v|^{q} \mathrm{~d} \mu\right)-\lambda\left(t_{1}\right) r\left(t_{1}\right) \\
& +\lambda\left(t_{1}\right) \int_{M} S|u|^{\alpha}|v|^{\beta} u v \mathrm{~d} \mu .
\end{aligned}
$$

The first term of the third line in result (52) is just $\lambda\left(t_{1}\right)$, so this term cancels with the second in that same line and what remains is exactly the desired result (47).

Theorem 4: Let $\left(M^{m}, g(t), \varphi(t)\right)$ be a solution of the Ricci harmonic flow on the smooth closed manifold $\left(M^{m}, g_{0}, \varphi_{0}\right)$ and $\lambda(t)$ denotes the evolution of the first eigenvalue under the flow. If $\kappa=\min \{p, q\}$ and

$$
\mathcal{S}-\frac{1}{\kappa} S g>0,
$$

on $M^{m} \times[0, T)$ with $S_{\min }(0)>0$. Then the quantity $\lambda(t)\left(1-\frac{2}{m} S_{\min }(0) t\right)^{m / 2}$ is nondecreasing along the flow on $[0, T)$ where $T=\min \left\{(m / 2) S_{\min }(0), T\right\}$.

Proof: It has been shown that 


$$
\begin{aligned}
\frac{\mathrm{d} \lambda}{\mathrm{d} t}(u, v, t)_{t=t_{1}}= & \lambda\left(t_{1}\right) \int_{M} S|u|^{\alpha}|v|^{\beta} u v \mathrm{~d} \mu \\
& +(\alpha+1) \int_{M} \mathcal{S}(\nabla u, \nabla u)|\nabla u|^{p} \mathrm{~d} \mu-\frac{\alpha+1}{p} \int_{M}|\nabla u|^{p} \mathrm{~d} \mu \\
& +(\beta+1) \int_{M} \mathcal{S}(\nabla v, \nabla v)|\nabla v|^{q} \mathrm{~d} \mu-\frac{\beta+1}{q} \int_{M}|\nabla v|^{q} \mathrm{~d} \mu .
\end{aligned}
$$

Using condition (53), the following bound is produced

$$
\begin{aligned}
\left.\frac{\mathrm{d} \lambda}{\mathrm{d} t}\right|_{t=t_{1}}> & \lambda\left(t_{1}\right) \int_{M} S|u|^{\alpha}|v|^{\beta} u v \mathrm{~d} \mu+\frac{\alpha+1}{\kappa} \int_{M} S|\nabla u|^{p} \mathrm{~d} \mu-\frac{\alpha+1}{p} \int_{M} S|\nabla u|^{p} \mathrm{~d} \mu \\
& +\frac{\beta+1}{\kappa} \int_{M} S|\nabla v|^{q} \mathrm{~d} \mu-\frac{\beta+1}{q} \int_{M} S|\nabla v|^{2} \mathrm{~d} \mu \\
= & \lambda\left(t_{1}\right) \int_{M} S|u|^{\alpha}|v|^{\beta} u v \mathrm{~d} \mu+(\alpha+1)\left(\frac{1}{\kappa}-\frac{1}{p}\right) \int_{M} S|\nabla u|^{p} \mathrm{~d} \mu \\
& +(\beta+1)\left(\frac{1}{\kappa}-\frac{1}{q}\right) \int_{M} S|\nabla v|^{q} \mathrm{~d} \mu .
\end{aligned}
$$

If $S_{\min }(0)>0$ then (41) implies that positivity of $S$ persists under this type of flow. Using (20) we have therefore,

$$
\begin{aligned}
\frac{\mathrm{d} \lambda}{\mathrm{d} t}(u, v, t)_{t=t_{1}} & \geq \lambda\left(t_{1}\right) \frac{S_{\min }(0)}{1-\frac{2}{m} S_{\min }(0) t_{1}} \int_{M}|u|^{\alpha}|v|^{\beta} u v \mathrm{~d} \mu \\
& =\lambda\left(t_{1}\right) \frac{S_{\min }(0)}{1-\frac{2}{m} S_{\text {min }}(0) t_{1}} .
\end{aligned}
$$

Then in any sufficiently small neighborhood about the value $t=t_{1}$, it can be concluded that

$$
\frac{\mathrm{d} \lambda}{\mathrm{d} t}(u, v, t) \geq \lambda(u, v, t) \frac{S_{\min }(0)}{1-\frac{2}{m} S_{\min }(0) t} .
$$

This is a separable equation, so integrating inequality (57) with respect to $t$ over the interval $\left[t_{0}, t_{1}\right] \subset I$,

$$
\int_{t_{0}}^{t_{1}} \frac{\mathrm{d} \lambda}{\lambda}>\int_{t_{0}}^{t_{1}} \frac{S_{\min }(0)}{1-\frac{2}{m} S_{\min }(0) t} \mathrm{~d} t .
$$

Integrating we obtain

$$
\log \left(\frac{\lambda\left(u\left(t_{1}\right), v\left(t_{1}\right), t_{1}\right)}{\lambda\left(u\left(t_{0}\right), v\left(t_{0}\right), t_{0}\right)}\right) \geq \log \left(\frac{1-\frac{2}{m} S_{\text {min }}(0) t_{1}}{1-\frac{2}{m} S_{\text {min }}(0) t_{0}}\right) .
$$

Since $\lambda\left(u\left(t_{1}\right), v\left(t_{1}\right), t_{1}\right)=\lambda\left(t_{1}\right)$ and $\lambda\left(u\left(t_{0}\right), v\left(t_{0}\right), t_{0}\right) \geq \lambda\left(t_{0}\right)$, it is concluded that

$$
\log \left(\frac{\lambda\left(t_{1}\right)}{\lambda\left(t_{0}\right)}\right) \geq \log \left(\frac{1-\frac{2}{m} S_{\min }(0) t_{1}}{1-\frac{2}{m} S_{\min }(0) t_{0}}\right)^{-m / 2}
$$


This implies that the function $\lambda(t)\left(1-\frac{2}{m} S_{\min }(0) t\right)^{m / 2}$ is nondereasing on any sufficiently small neighborhood of $t_{1}$. Since $t_{1}$ is arbitrary, we conclude that $\lambda(t)\left(1-\frac{2}{m} S_{\min }(0) t\right)^{m / 2}$ is nondecreasing along this flow over the interval $[0, T)$.

Now if $\alpha$ is taken to be zero, then the Ricci harmonic flow reduces to the Dirac flow and the theorem implies that the following Corollary can be stated.

Corollary 1: Let $g(t)$ for $t \in[0, T)$ be a closed Riemannian manifold $M^{m}$ such that $\lambda(t)$ denotes the first eigenvalue of the $(p, q)$-Laplacian With $\kappa=\min \{p, q\}$ and condition (53) in effect along the Ricci flow, then (a) If $R_{\min }(0) \geq 0$ then $\lambda(t)$ is nondecreasing along the Ricci flow for any $t \in[0, T)$. (b) If $R_{\min }(0)>0$ then the quantity $\left(1-R_{\min }(0) t\right) \lambda(t)$ is nondecreasing along the Ricci flow for any $t \in\left[0, T^{\prime}\right)$ where we define $T^{\prime}=\min \left\{T, 1 / R_{\min }(0)\right\}$.

If we simply work with two-dimensional manifolds or surfaces, then the following result must hold.

Theorem 5: Let $(g(t), \varphi(t))$ with $t \in[0, T)$ be a solution of the Ricci harmonic flow on a closed Riemannian surface $M^{m}$ and let $\lambda(t)$ denote the first eigenvalue of the $(p, q)$-Laplacian (8). (1) Suppose that $\kappa=\min \{p, q\}$ and Ric $-(R / \kappa) g \geq 0$ along the Ricci flow. If $S_{\text {min }}(0)>0$, the function $\left(1-S_{\min }(0) t\right) \lambda(t)$ is nondecreasing along the flow for any $t \in[0, T)$. If $S_{\text {min }}(0)>0$, the function $\left(1-S_{\text {min }}(0) t\right) \lambda(t)$ is nondecreasing along the Ricci-harmonic flow on $\left[0, T^{\prime}\right)$ where $T^{\prime}=\min \left(T^{\prime}, 1 / S_{\text {min }}(0)\right)$. (2) Suppose that $|\nabla \varphi|^{2} \geq \kappa \nabla \varphi \otimes \nabla \varphi$. If $S_{\text {min }}(0) \geq 0$ then $\lambda(t)$ is nondecreasing along the Ricci-harmonic flow for any $t \in[0, T)$. If $S_{\min }(0)>0$ the quantity

$\left(1-S_{\min }(0) t\right) \lambda(t)$ is nondecreasing along this flow on $\left[0, T^{\prime}\right)$ where $T^{\prime}=\min \left\{T^{\prime}, 1 / S_{\min }(0)\right\}$.

Proof: In the case of two dimensional manifolds, the tensor Ric takes the simple form,

$$
R_{i j}=\frac{R}{2} g_{i j} .
$$

Consequently, we can calculate that

$$
\begin{aligned}
\mathcal{T}_{i j} & =\mathcal{S}_{i j}-\frac{S}{\kappa} g_{i j}=\frac{R}{2} g_{i j}-\alpha \nabla_{i} \varphi \nabla_{j} \varphi-\frac{1}{\kappa}\left(R-\alpha|\nabla \varphi|^{2}\right) g_{i j} \\
& =\left(\frac{1}{2}-\frac{1}{\kappa}\right) R g_{i j}-\alpha \nabla_{i} \varphi \nabla_{j} \varphi+\frac{\alpha}{\kappa}|\nabla \varphi|^{2} g_{i j} .
\end{aligned}
$$

For any vector $w$, then we can contract $\mathcal{T}_{i j}$ with the $w^{i}$ to get

$$
\begin{aligned}
\mathcal{T}_{i j} w^{i} w^{j} & =\left(\frac{1}{2}-\frac{1}{\kappa}\right) R|w|^{2}-\alpha\left(\nabla_{i} \varphi w^{i}\right)^{2}+\frac{\alpha}{\kappa}|\nabla \varphi|^{2}|w|^{2} \\
& \geq\left(\frac{1}{2}-\frac{1}{\kappa}\right) R|w|^{2}+\alpha\left(\frac{1}{\kappa}-1\right)|\nabla \varphi|^{2}|w|^{2} .
\end{aligned}
$$

If Ric $\geq \varepsilon \nabla \varphi \otimes \nabla \varphi$ where $\varepsilon \geq 2 \alpha(\kappa-1) /(\kappa-2)$, then $R \geq \varepsilon|\nabla \varphi|^{2}$, hence 
using $\varepsilon$ and simplifying, we have

$$
\mathcal{T}_{i j} w^{i} w^{j} \geq\left[\left(\frac{1}{2}-\frac{1}{\kappa}\right) \varepsilon+\alpha\left(\frac{1}{\kappa}-1\right)\right]|\nabla \varphi|^{2}|w|^{2} \geq 0 .
$$

Now with $\mathcal{S}_{i j}=\operatorname{Ric}_{i j}-\alpha \nabla_{i} \varphi \nabla_{j} \varphi$ and $S=R-\alpha|\nabla \varphi|^{2}$,

$$
\begin{aligned}
\mathcal{T}_{i j} w^{i} w^{j} & =\mathcal{S}_{i j} w^{i} w^{j}-\frac{S}{\kappa}|w|^{2} \\
& =\operatorname{Ric}_{i j} w^{i} w^{j}-\alpha\left(\nabla_{i} \varphi w^{i}\right)^{2}-\frac{1}{\kappa}\left(R-\alpha|\nabla \varphi|^{2}\right)|w|^{2} \\
& \geq R_{i j} w^{i} w^{j}-\frac{\alpha}{\kappa}|\nabla \varphi|^{2}|w|^{2}-\frac{R}{\kappa}|w|^{2}+\frac{\alpha}{\kappa}|\nabla \varphi|^{2}|w|^{2} \\
& =R_{i j} w^{i} w^{j}-\frac{R}{\kappa}|w|^{2}=\left(\frac{1}{2}-\frac{1}{\kappa}\right) R|w|^{2} \geq 0 .
\end{aligned}
$$

To get the second last inequality in (65), use has been made of $R_{i j}$ in (61) for the two-dimensional case. The result now follows by using Theorems 2 and 4 .

Corollary 2: Let $g(t), t \in[0, T)$ be a solution of the Ricci flow on a closed Riemannina surface $M^{m}$ and $\lambda(t)$ denotes the first eigenvalue of the $(p, q)$ Laplacian (8). (1) If $R_{\min }(0) \geq 0$ then $\lambda(t)$ is nondecreasing along the Ricci flow for any $t \in[0, T)$. (2) If $R_{\min }(0)>0$, then the quantity $\left(1-R_{\min }(0) t\right) \lambda(t)$ is nondecreasing along the Ricci flow for any $t \in\left[0, T^{\prime}\right)$ where $T^{\prime}=\min \left\{T^{\prime}, 1 / R_{\min }(0)\right\}$

As an illustration of these ideas, let $\left(M^{m}, g_{0}\right)$ be an Einstein manifold so there exists a constant $\beta$ such that $\operatorname{Ric}\left(g_{0}\right)=\beta g_{0}$ and suppose $\left(N^{n}, \sigma\right)=\left(M^{m}, g_{0}\right)$ so $\varphi_{0}$ is the identity map. Assuming that $g(t)=c(t) g_{0}$, $c(0)=1$ is a function and the fact $\varphi(t)=\varphi(0)$ is a harmonic map for all $g(t)$, then the Ricci-harmonic flow reduces to

$$
\frac{\partial c(t)}{\partial t}=-2 \beta+2 \alpha, \quad c(0)=1 .
$$

The solution for $c(t)$ of the initial value problem is given by

$$
c(t)=(-2 \beta+2 \alpha) t+1 \text {. }
$$

The solution of the flow remains Einstein and so we have,

$$
\begin{aligned}
& \mathcal{S}=\operatorname{Ric}_{g(t)}-\alpha \nabla \varphi \otimes \nabla \varphi=(\beta-\alpha) g_{0}=\frac{\beta-\alpha}{-2(\beta-\alpha) t+1} g(t), \\
& S=R-\alpha|\nabla \varphi|^{2}=\frac{(\beta-\alpha) m}{-2(\beta-\alpha) t+1} .
\end{aligned}
$$

By (46), we find that

$$
\frac{\mathrm{d} \lambda}{\mathrm{d} t}(u, v, t)_{t=t_{1}}=\frac{\beta-\alpha}{-2(\beta-\alpha) t+1}\left((\alpha+1) \int_{M}|\nabla u|^{p} \mathrm{~d} \mu+(\beta+1) \int_{M}|\nabla v|^{q} \mathrm{~d} \mu\right) .
$$

If it is assumed that $p \leq q$ then for $\alpha \leq \beta$ and $t_{1} \in\left[0, T^{\prime \prime}\right)$ where $T^{\prime \prime}=\min \{1 /(2(\beta-\alpha)), T\}$, we have

$$
\frac{\mathrm{d} \lambda}{\mathrm{d} t}(u, v, t)_{t=t_{1}} \geq \frac{\beta-\alpha}{-2(\beta-\alpha) T_{1}+1} \lambda\left(t_{1}\right) .
$$


In any sufficiently small neighborhood of $t_{1}$,

$$
\frac{\mathrm{d} \lambda}{\mathrm{d} t} \geq \frac{\beta-\alpha}{-2(\beta-\alpha) t+1} \lambda(u, v, t) .
$$

Integrating this inequality with respect to $t$ on $\left[t_{0}, t_{1}\right]$, we find that

$$
\ln \frac{\lambda\left(u\left(t_{1}\right), v\left(t_{1}\right), t_{1}\right)}{\lambda\left(u\left(t_{0}\right), v\left(t_{0}\right), t_{0}\right)} \geq \ln \left(\frac{-2(\beta-\alpha) t_{1}+1}{-2(\beta-\alpha) t_{0}+1}\right)^{-p / 2} .
$$

As $t_{1} \in\left[0, T^{\prime \prime}\right)$ is arbitrary, $\lambda\left(u\left(t_{1}\right), v\left(t_{1}\right), t_{1}\right)=\lambda\left(t_{1}\right)$ and $\lambda\left(u\left(t_{0}\right), v\left(t_{0}\right), t_{0}\right) \geq \lambda\left(t_{0}\right)$. It can be concluded from this that $\lambda(t)(-2(\beta-\alpha) t+1)^{p / 2}$ is nondecreasing along the Ricci-harmonic flow on $\left[0, T^{\prime \prime}\right)$.

\section{Summary}

The main results here have been to define a $p$-Laplacian eigenvalue problem and to find a way to study the evolution of the first eigenvalue under the Ricci flows established in Equations (2) and (3). It has also been found that flows for some related quantities can also be studied. This work will provide a foundation for the study of similar problems in the future.

\section{Conflicts of Interest}

The author declares no conflicts of interest regarding the publication of this paper.

\section{References}

[1] Perlman, G. (2002) The Entropy Formula for the Ricci Flow and Its Geometric Applications. ArXiv:0211159.

[2] Yano, K. (1970) Integral Formulas in Riemannian Geometry. Marcel Dekker, NY.

[3] Bracken, P. (2017) An Introduction to the Ricci Flow for Two-Dimensional Manifolds, Advances in Mathematics Research. Nova Science Publishers, Hauppauge, NY, 155-163.

[4] Morita, S. (2001) Geometry of Differential Forms. AMS Translations of Mathematical Monographs, 201. https://doi.org/10.1090/mmono/201

[5] Aubin, T. (2001) A Course in Differential Geometry. AMS Graduate Studies in Mathematics, 27. https://doi.org/10.1090/gsm/027

[6] Cao, X.-D. (2007) Eigenvalues of $(-\Delta+R / 2)$ on Manifolds with Nonnegative Curvature Operator. Mathematische Annalen, 337, 435-441. https://doi.org/10.1007/s00208-006-0043-5

[7] Cao, X.-D. (2008) First Eigenvalues of Geometric Operators under the Ricci Flow. Proceedings of the American Mathematical Society, 136, 4075-4078. https://doi.org/10.1090/S0002-9939-08-09533-6 